\title{
Zebrafish Danio rerio shows behavioural cross- context consistency at larval and juvenile stages but no consistency between stages
}

\author{
Alfonso Sébastien ${ }^{1,2,{ }^{*}}$, Peyrafort Manon ${ }^{1}$, Cousin Xavier ${ }^{2,3}$, Bégout Marie-Laure 1,2
}

\author{
1 Ifremer, Laboratoire Ressources HalieutiquesPlace Gaby Coll F-17137 L'Houmeau, France \\ 2 MARBEC, Univ. Montpellier CNRS, Ifremer, IRD Chemin de Maguelone F-34250 Chemin de \\ Maguelone Palavas-les-flots, France \\ 3 Université Paris-Saclay, INRAE, AgroParisTech, GABI Domaine de Vilvert Bâtiment 231, F-78350 \\ Jouy-en-Josas, France \\ ${ }^{4}$ MARBEC, Univ. Montpellier CNRS, Ifremer, IRD Chemin de Maguelone F-34250 Chemin de \\ Maguelone Palavas-les-flots, France
}

*Corresponding author : Sébastien Alfonso, email address : sebastien.alfonso1@gmail.com

\begin{abstract}
:
Coping style is defined as a set of individual physiological and behavioural characteristics consistent across time and context. In the zebrafish Danio rerio, as well as in many other animals, several covariations have been established between behavioural, physiological, and molecular responses. However, not many studies have addressed the consistency of behavioural responses over time starting at the larval stage. Therefore, we aimed to improve our understanding of behavioural consistency across context and over time in zebrafish from the larval to juvenile stages. We conducted two distinct experiments: a larval stage experiment (from 8 to 21 days post fertilization, dpf) and a juvenile stage experiment (from 21 to $60 \mathrm{dpf}$ ). On one hand, the larval experiment allows to focus on the transition between 8 and $21 \mathrm{dpf}$, marked by significant morphological changes related to the end of larval stage and initiation of metamorphosis. On the other hand, the juvenile experiment allows to properly cover the period extending from the end of larval stage to the juvenile stage (60 dpf) including metamorphosis which is itself completed around $45 \mathrm{dpf}$. Within each experiment, boldness was determined using a group risk-taking test to identify bold and shy individuals. A novel environment test was then performed at the same age to evaluate consistency across context. Groups of fish (either bold or shy) were bathed in an alizarin red $\mathrm{S}$ solution for later identification of their initially determined coping style to evaluate behavioural consistency over time. Fish were then reared under common garden conditions and challenged again with the same behavioural tests at a later age (21 and $60 \mathrm{dpf}$ in the larval and juvenile experiments, respectively). We observed behavioural consistency across context, with bold fish being more active and expressing higher thigmotaxis regardless of age. There was, however, little behavioural consistency over age, suggesting behavioural plasticity during development. Moreover, the use of alizarin red $S$ to conduct this experiment provides new perspectives for the further study of the longitudinal evolution of various traits, including behaviour, over life stages in fish.
\end{abstract}

Keywords : coping style, fish, personality, vital staining 


\section{1 | INTRODUCTION}

Coping style is defined as a coherent set of individual physiological and behavioural traits that are consistent across context and over time, i.e. in different situations and at different times (Koolhaas et al., 1999). The existence of coping styles has been demonstrated in many animal species, including fish, and are described as a continuum between two extreme phenotypes, which are often called proactive and reactive (Øverli et al., 2007; Tudorache et al., 2013; Ferrari et al., 2015; Castanheira et al., 2017; Wong et al., 2019). Temperament (Reale et al., 2007), personality (Gostling, 2001; Reale et al., 2010), and behavioural syndrome (Sih et al., 2004) are terms related to coping style, as defined by Koolhaas et al. (1999). In fish, proactive individuals are generally bolder (Øverli et al., 2006; Huntingford et al., 2010; Alfonso et al., 2019b), more aggressive (Øverli et al., 2004), more active (Øverli et al., 2002), and explore their environment faster than reactive fish (Millot et al., 2009; Ferrari et al., 2015).

Behavioural consistency across context and over time has been shown in adult fish, including the zebrafish Danio rerio (Rey et al., 2013; Toms and Echevarria, 2014). For example, boldness, aggression, fear, and exploration, measured in five distinct contexts, were shown to be consistent over a period of seven days (Toms and Echevarria, 2014). Rey et al. (2013) also showed that boldness, measured in a risk-taking test, was positively correlated with aggressive behaviour and highly consistent over 10 months in adults. In most cases, including those mentioned above, coping styles were however studied in juveniles or adults and consistency over time was generally evaluated through repeated assays within a single developmental stage. Coping styles have been, however, occasionally determined at early 
stages, i.e. larval stages in fish. Tudorache et al. (2015) showed that swimming behaviour differed in the larvae of zebrafish eight days post fertilization (dpf), depending on the order of emergence from a sheltered compartment. Early emerging larvae displayed lower velocity and travelled less distance than later emerging ones. Moreover, early emerging larvae expressed higher cortisol levels in response to netting stress but reached baseline level faster than late emerging larvae. Pasquet et al. (2016) showed that northern pike Esox lucius larvae exhibited consistency in behavioural responses across contexts in maze and novel object tests. More active larvae visited a higher number of zones in the maze and spent more time near the novel object than others, indicating that coping styles were present and consistent across context at the larval stage. Such cross-context consistency at early stage is contradictory to observations of the mangrove killifish Kryptolebias marmoratus, in which it was absent before the juvenile stage at 61 days post hatching (Edenbrow and Croft, 2011).

Ontogenic studies evaluating the consistency of coping style across life stages in fish are scarce. The consistency of coping style between early (likely larval) and later stages has only been evaluated in two studies using two species, mangrove killifish and nine-spine sticklebacks Pungitius pungitius, and the authors concluded that there was no behavioural consistency between these life stages (Edenbrow and Croft, 2011; Herczeg et al., 2013). However, in both studies, individuals were kept isolated to allow for later individual identification. Such a rearing method can introduce several biases, such as divergence in physico-chemical variables, unintentional differences in feeding (especially with such small individuals), or stress induced by social isolation (e.g. in zebrafish, Pagnussat et al. (2013)). 
An option to circumvent these limitations is batch marking of larvae. Indeed, this method allows the rearing of fish, from different coping styles, under common garden conditions. Alizarin red $\mathrm{S}\left(\mathrm{C}_{14} \mathrm{H}_{7} \mathrm{NaO}_{7} \mathrm{~S}\right)$ is a fluorescent dye that labels calcium-containing tissue and has thus been used to monitor skeletal mineralization in vivo in zebrafish (DeLaurier et al., 2010; Tu and Johnson, 2011; Bensimon-Brito et al., 2016). Recently, alizarin red S was successfully used for mass marking of several fish species at an early stage, showing no particular mortality or growth delay (Caraguel et al., 2015; Lü et al., 2015; Stanczak et al., 2015). The mass marking of larvae can be performed by ingestion of Artemia salina previously bathed in an alizarin red S solution (Stanczak et al., 2015) or by directly bathing the larvae in the solution (Caraguel et al., 2015; Lü et al., 2015). Thus, alizarin red S appears to be a promising non-invasive tool to mark larvae and monitor behavioural consistency in zebrafish across life stages. Such information is indeed essential for understanding how development and/or environment may shape behaviour and coping styles.

Thus, the objectives of this study were to evaluate behavioural consistency across contexts and over time in zebrafish from the larval to juvenile stage. Zebrafish generally hatch at 2-3 $\mathrm{dpf}$ and reach the juvenile stage at $45 \mathrm{dpf}$ for finally being considered as adults around $90 \mathrm{dpf}$ when reared at $28^{\circ} \mathrm{C}$ (Nüsslein-Volhard and Dahm, 2002; Singleman and Holtzman, 2014; Westerfield, 2000). Metamorphosis is the continuous process leading from larvae to juvenile. It has no absolute borders because it depends on the analysed trait and also because metamorphosis course depends on external factors such as temperature or food availability (Parichy 2009). As an example, skin pigmentation pattern changes from around 14 until around $30 \mathrm{dpf}, 22 \mathrm{dpf}$ being defined as the middle of metamorphosis (Parichy and Turner 
2003). The age of 21-22 dpf appeared to be pivotal for other physiological or morphological changes such as the end of the transition from skin to gill respiration (Hale, 2014), and the start of gonadal transformation from the "juvenile ovary" stage (Orban et al., 2009). This age is also marked by changes in neurobehavioral abilities (Valente et al., 2012). These developmental steps shaped the rationale of our experiment and we decided to conduct two distinct experiments, a so-called 'larval experiment' from 8 to $21 \mathrm{dpf}$ and a 'juvenile experiment' from 21 to $60 \mathrm{dpf}$. On one hand, the larval experiment allows to focus on the transition between 8 and $21 \mathrm{dpf}$, marked by significant morphological changes related to the end of larval stage and the initiation of metamorphosis. On the other hand, the juvenile experiment allows to properly cover the period extending from the end of larval stage to the juvenile stage (60 dpf) including metamorphosis.

Within each experiment, fish were screened for being bold or shy using a group risk-taking test and thereafter challenged in an individual novel environment test at the same age to first evaluate consistency across context. Second, behavioural consistency over time was monitored by comparing fish behavioural responses between the two successive ages (i.e., between 8 and 21 and between 21 and $60 \mathrm{dpf}$ ) using alizarin red S marking for coping-style identification.

\section{2 | MATERIALS AND METHODS}


This study was conducted with the approval of the Animal Care Committee of Poitou-138 Charentes \# 84 COMETHEA (France) under project authorization number CE2012-23 and followed the recommendations of Directive 2010/63/EU.

\section{1 | Fish rearing}

The study was conducted on the zebrafish TU strain (ZFIN ID: ZDBGENO-990623-3), maintained at the Fish Ecophysiology Platform (PEP - http://wwz.ifremer.fr/pep_eng) and originating from the European Zebrafish Resources Center (EZRC, Karlsruhe, Germany).

Eggs were obtained by random pairwise mating using adults from our stock at approximately 180 dpf to provide larvae for the behavioural experiments. Briefly, one male and one female were placed together in spawning boxes on the evening before the eggs were required (AquaSchwarz, Germany). Eggs were collected the following morning and the fertilization rate assessed within $2 \mathrm{~h}$ of collection. Spawns with a fertilization rate $>80 \%$ were kept for experiments and placed in Petri dishes filled with $30 \mathrm{~mL}$ isotonic medium E3 (1 L: $17.2 \mathrm{~g}$ NaCl, 0.76 g HCl, 2.9 g CaCl$\left._{2} .2 \mathrm{H}_{2} \mathrm{O}, 4.9 \mathrm{~g} \mathrm{MgSO}_{4} .7 \mathrm{H}_{2} \mathrm{O}\right)$ and methylene blue (250 $\mu \mathrm{L}^{\mathrm{L}} \mathrm{L}^{-1}$ ) and incubated at $28^{\circ} \mathrm{C}$ under a $14-\mathrm{h} / 10$-h dark/light cycle as described in (Alfonso et al., 2019a). The next day, 10 eggs from six different spawns were mixed to avoid spawn-effects to provide 60 embryos for one Petri dish. Multiple Petri dishes were prepared, depending on the number of replicates needed. At 72 h post fertilization (hpf), after hatching, the chorions were removed from the petri dishes and the larvae returned to the incubator until $5 \mathrm{dpf}$. They were then transferred from Petri dishes to 1L tanks. Initial behavioural tests and alizarin red S marking were performed at 8 and 21 dpf. After these first characterization and marking steps, larvae with both coping styles were maintained in a common garden, as explained below. 
Larvae were reared in $1 \mathrm{~L}$ tanks from 8 to $21 \mathrm{dpf}$ ( 24 larvae per tank, $\mathrm{n}=12$ per coping style) and at $21 \mathrm{dpf}$, they were transferred to 10L tanks in the rearing system (20 larvae per tank, $\mathrm{n}=$ 10 per coping style). They were reared under a 14-h/10-h dark/light cycle in both cases. Physical water conditions were maintained constant during the experiment: temperature of 27 $\pm 1^{\circ} \mathrm{C}$, conductivity of $300 \pm 50 \mu \mathrm{S} . \mathrm{cm}^{-1}$, and $\mathrm{pH} 7.5 \pm 0.5$, using a mixture of reverse osmosis water and tap water, both initially treated with charcoal and sediment filters. Ammonia, nitrites, and nitrates were monitored weekly and remained within recommended ranges (Lawrence, 2007). Fish were fed three times a day, i.e. morning and evening with pellets and at midday with freshly hatched Artemia salina. The size of the food pellets was adapted to the age of the fish and size of the mouth. From $5 \mathrm{dpf}$ to the end of experiment, fish were fed sequentially with $100 \mu \mathrm{m}, 200 \mu \mathrm{m}$, and $300 \mu \mathrm{m}$ SDS (Special Diet Service; Dietex international), according to the size of the fish.

\section{2 | Experimental procedure to monitor behavioural consistency across contexts and} over age

Two experiments, using different fish, were performed covering the larval stage (Larval experiment; from 8 to $21 \mathrm{dpf}$ ) and part of the juvenile stage (Juvenile experiment; from 21 to $60 \mathrm{dpf}$ ); the steps are summarised in Figure 1. In both cases, the first step consisted of a risktaking test performed in groups at $8 \mathrm{dpf}$ (Larval experiment) and $21 \mathrm{dpf}$ (Juvenile experiment) to measure boldness and thus assess coping style. Individuals were then kept isolated overnight. The following day, a novel environmental test was performed to evaluate behavioural consistency across contexts (see details in the "Behavioural experiments" section). After the second test, fish exhibiting one or the other coping style were bathed in 
vital stain using alizarin red $\mathrm{S}\left(\mathrm{C}_{14} \mathrm{H}_{7} \mathrm{NaO}_{7} \mathrm{~S}\right.$; see details in the "Marking procedure" section) and fish of both coping styles further reared under common conditions garden to avoid possible rearing bias or stress from isolation. Later, at $21 \mathrm{dpf}$ for the larval experiment and $60 \mathrm{dpf}$ for the juvenile experiment, the fish were again challenged to evaluate behavioural consistency over life stages and alizarin red S marking was read to determine the initial coping style.

\section{3 | Behavioural experiments}

All behavioural experiments were performed in the experimental room at $27^{\circ} \mathrm{C}$, with a photoperiod synchronized to that of the rearing room, i.e. $14 \mathrm{~h} / 10 \mathrm{~h}$ (light/dark). After each experiment, the water was changed. The fish were fed once on the day of the experiment at the end of the day.

Videos for the larval stage behavioural experiments (8 or $21 \mathrm{dpf}$ ) were recorded using DanioVision (Noldus, The Netherlands). Videos for the juvenile stage (60 dpf) behavioural experiments were recorded using an ICD-48E analogue camera (Ikegami, Japan) and a 2.113.5 lens (Fujinon, Japan) linked to a computer with an acquisition card and EthoVision XT 10.1 software (Noldus, The Netherlands). Data extraction and analyses were performed using EthoVision XT 10.1 software.

\subsection{1 | Boldness screening using group risk-taking tests}

The day before conducting the test, groups of fish were gently caught from their home tank and groups of 15 individuals assembled. The groups of fish were placed in a Petri dish (90 $\mathrm{mm}$ in diameter $\mathrm{x} 14.2 \mathrm{~mm}$ in height) at both 8 and $21 \mathrm{dpf}$ or in a 3L tank at $60 \mathrm{dpf}$ and then 
transferred to the experimental room for acclimation. The next day, the group risk-taking test was performed to assess boldness. Risk-taking tests were performed on 22 groups $(\mathrm{n}=330$ larvae) at $8 \mathrm{dpf}$ and four groups ( $=60$ larvae) at $21 \mathrm{dpf}$ for the larval experiment. For the juvenile experiment, the risk-taking tests were performed on seven groups ( $\mathrm{n}=105$ larvae) at $21 \mathrm{dpf}$ and five groups ( $\mathrm{n}=50 \mathrm{fish})$ at $60 \mathrm{dpf}$.

Three different risk-taking devices were used depending on the age of the fish. At 8 dpf, the device consisted of two Petri dishes (3.3 $\mathrm{cm}$ in diameter $\mathrm{x} 1 \mathrm{~cm}$ in height) connected to each other by a passage tube $(0.5 \mathrm{~cm}$ in diameter x $0.6 \mathrm{~cm}$ in height, Tudorache et al. (2015)). One Petri dish was covered with black tape, whereas the other one was not. At $21 \mathrm{dpf}$, the device was composed of a dark, covered compartment ( $4 \mathrm{~cm}$ x $7 \mathrm{~cm}$ x $2.5 \mathrm{~cm}$ in height) connected by an opening situated at the centre of the connecting face of the compartment to another one that was lit (6.5 cm x $7 \mathrm{~cm} \times 2.5 \mathrm{~cm}$ in height). At $60 \mathrm{dpf}$, the device was composed of a dark, covered compartment ( $8 \mathrm{~cm} \times 10 \mathrm{~cm} \times 11 \mathrm{~cm}$ in height) connected by an opening $(2.5 \mathrm{~cm}$ in diameter) situated at the centre of the connecting face of the compartment to another one that was lit (15 cm x $10 \mathrm{~cm}$ x $11 \mathrm{~cm}$ in height) (Rey et al., 2013).

Regardless of age, the risk-taking test started by gently introducing the group of fish $(n=15)$ into the sheltered compartment. After an acclimation period of $15 \mathrm{~min}$, a door was opened, allowing fish to exit the sheltered compartment to enter the risky lit compartment for a period lasting $10 \mathrm{~min}$. At the end of the experiment (i.e. 10 min after opening the door) or after half of the fish had left the sheltered area (if less than $10 \mathrm{~min}$ ), the connection between the two compartments was closed. The 10 min duration was used based on the study of Tudorache et al. (2015). Larvae (8 or $21 \mathrm{dpf}$ ) were gently caught using a $3 \mathrm{~mL}$ pipette (with a wide-cut 
opening) and placed individually into a Petri dish (3.3 cm in diameter x $2.5 \mathrm{~cm}$ in height). At $60 \mathrm{dpf}$, fish were netted and placed individually in a 1L tank (AquaSchwarz, Germany) before further behavioural experiments. Fish that left the sheltered compartment were considered to be bold and fish that stayed in the covered compartment were considered to be shy. The proportion of bold and shy fish (\%) was calculated for each group and age.

\subsection{2 | Individual open-field test and novel tank diving test}

The day after the risk-taking test, fish were tested individually to evaluate behavioural consistency across contexts and identify a behavioural syndrome by measuring activity and thigmotaxis/vertical positioning ( 8 dpf: $n=48$ bold, $n=48$ shy; 21 dpf: $n=50$ bold, $n=55$ shy; 60 dpf: $n=24$ bold, $n=23$ shy). Depending on the age of the fish, three different devices and experimental procedures were used, but all three belonged to the family of novel environment test devices, either open-field or novel-tank diving tests..

The open-field test for 8 dpf larvae was adapted from Tudorache et al. (2015). Larvae were individually transferred into one well of a 24-well plate (Krystal 24, opaque wall and clear bottom microplate) and placed into the DanioVision device. After an acclimation period of 5 min in darkness, the light was switched on and a video recording made in top view for 10 min. The open-field arena was virtually separated into two areas of equal volume (centre and periphery), according to Schnorr et al. (2012). The distance travelled (cm) and the proportion of time spent in the peripheral half (\%) were calculated to evaluate activity and thigmotaxis, respectively. At 21 dpf, the open-field test was adapted from Ahmad and Richardson (2013). Larvae were individually transferred to a Petri dish (3.3 cm in diameter x $1 \mathrm{~cm}$ in height) containing $5 \mathrm{~mL}$ E3 medium and placed into the DanioVision device. A video was recorded 
in top view for $10 \mathrm{~min}$. the open-field arena was virtually separated into two areas of equal volume (centre and periphery). The distance travelled $(\mathrm{cm})$ and the proportion of time spent in the peripheral half (\%) were recorded to evaluate activity and thigmotaxis, respectively. At 60 dpf, a novel tank diving test adapted from Egan et al. (2009) was used. Fish were individually transferred into a novel tank (trapezoidal 1.5 L tank, Aquatic Habitats) and a video recording was made in side view for $4 \mathrm{~min}$. The novel-tank arena was virtually separated into areas of two equal volumes (top and bottom) according to Egan et al. (2009). The distance travelled (cm) and the proportion of time spent in the bottom half (\%) were recorded to evaluate activity and bottom dwelling, respectively.

\section{4 | Marking procedure}

After behavioural characterisation (i.e. at 8 or $21 \mathrm{dpf}$ for the larval and juvenile experiments, respectively) groups of fish identified as either bold or shy, were marked using alizarine red S and the procedure described below (section 2.4). Thus, either bold or shy marked fish were reared under common garden conditions (i.e. in a common tank) with unmarked larvae of the opposite coping-style until the next assessment (see Figure 1).

\section{4 | Vital staining by alizarin red S, reading procedure, and LPMR test}

This section presents the marking procedure used for the experiment described above, as well as the preliminary experiments carried out to validate the use of alizarin red $\mathrm{S}$ as a vital stain.

\subsection{1 | Marking procedure}

Alizarin red $\mathrm{S}$ is a fluorochrome certified by the Biological Stain Commission for in vivo marking. We validated the marking procedure of Bensimon-Brito et al. (2016) by first 
verifying that marking had no effect on development, survival, or larval behaviour by comparing these features between control and alizarine red S marked larvae. Triplicates $(n=3$ x 40 larvae) were assembled for each treatment for both larval and juvenile experiments. Treated larvae were bathed in the $1 \mathrm{~L}$ system in water containing $0.01 \%$ alizarin red S (SigmaAldrich, St. Quentin Fallavier, France) buffered with sodium hydroxide (50 $\mu \mathrm{L}$ of $0.5 \mathrm{M}$ $\mathrm{NaOH}$ ) for $150 \mathrm{~min}$. After the bathing period, larvae were rinsed in clean water twice before reintroduction into the rearing tank. Larvae marked at $8 \mathrm{dpf}$ were then observed at $21 \mathrm{dpf}$ (Larval experiment) and larvae marked at $21 \mathrm{dpf}$ were observed at $60 \mathrm{dpf}$ (Juvenile experiment) to verify the efficiency of the marking protocol. The control groups were treated in the same way, except they were not exposed to alizarine red S, and were reared in separate tanks under the same conditions. Survival and persistence of the staining were monitored up to the end of the test period.

\subsection{2 | Reading procedure}

Larvae or juveniles were anaesthetized with benzocaine $\left(50 \mu\right.$ of a stock solution $\left(50\right.$ g.L $\mathrm{L}^{-1}$ in 100\% ethanol); Sigma-Aldrich, St. Quentin Fallavier, France) for vital staining reading using a stereo microscope (Olympus SZX9) under green fluorescent light (510-550 nm, X-Cite, 120Q EXFO). Images were captured using a camera (DFX31AU03, The Imaging Source, Germany) and IC Capture 2.4 software (The Imaging Source, Germany).

\subsubsection{Monitoring of larval behaviour after alizarin red S marking}

To ascertain that the marking procedure has no behavioural effects, the larval photomotor response (LPMR) test was performed (Schnorr et al. (2012); Vignet et al. (2015)) on a subsample of larvae ( $\mathrm{n}=30$ per treatment). The day after alizarin red $\mathrm{S}$ marking (i.e. $9 \mathrm{dpf}$ ), 
larvae were transferred individually into the wells of 24-well plates (Krystal 24, opaque wall and clear bottom microplate) for acclimation and the plates transferred to the experimental room, as described above. An equal number of larvae from each treatment were introduced into each plate to avoid a potential trial effect. Plates were successively placed into a DanioVision device. After an initial 10-min acclimation period in the DanioVision device, the LPMR was performed, consisting of the following 5-min steps: Light on-1 (LON1, 70 lux), Light off (LOFF, < 1 lux), and Light on-2 (LON2, 70 lux), with constant infra-red light maintained during video recording. The distance travelled $(\mathrm{cm})$ was recorded and summed for each 5-min step.

\subsection{Statistical analyses}

Statistical analyses were performed using R 3.1.0 software (R Core Team, 2013). All statistical analyses were carried out at a 95\% level of significance. Values are represented as the mean \pm SE. Normality of the data was verified using the Shapiro test before applying either parametric (ANOVA) or non-parametric tests (Wilcoxon rank sum test), depending on whether normality was verified or not.

The proportion of fish that left the covered area (bold) during the risk-taking tests were compared between ages using the chi-squared test, followed by pairwise multiple comparisons. P-values were adjusted using the Holm method.

Principal component analysis (PCA) was performed within each age group for the open-field and novel tank diving tests using the informative variables for activity (the distance travelled (cm)) and thigmotaxis orvertical positioning (i.e., and proportion of time spent in the 
peripheral area (\%) at 8 and $21 \mathrm{dpf}$ and the proportion of time spent in the bottom area (\%) at 60 dpf) using the ade4 package (Dray and Dufour, 2007). Individual PC scores were downloaded for the first axis (PCA axis 1) to extract individual behavioural scores, as described in numerous studies (Castanheira et al., 2013; Millot et al., 2014; Ferrari et al., 2015; Castanheira et al., 2016). Consistency across contexts [Risk taking vs. open field/novel tank diving tests] was estimated using the Wilcoxon rank sum test on individual behavioural scores to compare coping styles (i.e. bold vs shy according to the risk-taking of the corresponding age) within each age group of the two experiments.

Finally, the exact binomial test was performed to evaluate behavioural consistency in the risktaking test between two successive ages (i.e., between 8 and $21 \mathrm{dpf}$ and 21 and $60 \mathrm{dpf}$ ) with random theoretical probability within each coping style $(\mathrm{p}=0.5$, i.e. the equal probability of being characterized as bold or shy in the second test). The chi-squared test was also performed to compare behavioural consistency in risk taking between coping styles within each experiment.

In parallel, survival was compared between bathed and control larvae using the log-rank Mantel-Cox test to confirm the harmlessness of vital staining. For the LPMR test, the distance travelled was compared between treatments (Control vs Marked) with a repeated-measures ANOVA (with 3 periods, i.e. LON-1, LOFF, LON-2) followed by Tukey’s HSD post-hoc test.

\section{3 | RESULTS}




\section{1 | Validation of the alizarin red S marking procedure}

In preliminary test groups, $100 \%$ of larvae bathed in $0.01 \%$ alizarin red S for $150 \mathrm{~min}$ at 8 and $21 \mathrm{dpf}$ showed visible marks in the otolith and calcified tissues of the caudal peduncle at 21 and 60 dpf, respectively (Figure 2a,b). Survival in the stained groups was above $80 \%$ and identical to that of the control groups (Mantel Cox: $\chi^{2}=1.470, \mathrm{df}=1, \mathrm{p}=0.23$ and $\chi^{2}=0.005$, $\mathrm{d} \mathrm{f}=1, \mathrm{p}=0.94$ for marking at 8 and $21 \mathrm{dpf}$, respectively).

The distance travelled in the LPMR test was higher during the LOFF than LON1 and LON2 periods, regardless of the treatment (control vs marked) (repeated measures ANOVA, $\mathrm{df}=2$, $\mathrm{F}=72.5, \mathrm{p}<0.001)$ and there was no effect of treatment $(\mathrm{F}=0.4$, df $=3, \mathrm{p}=0.5)$ or interaction between treatment and period $(\mathrm{F}=0.1, \mathrm{df}=3, \mathrm{p}=0.9$; Figure 3 ).

\section{2 | Risk-taking test}

The proportion of bold fish, individuals which left the covered compartment during the risktaking test, was significantly different between ages $\left(\chi^{2}=62.7\right.$, $\mathrm{df}=3, \mathrm{p}<0.001$; Figure 4). Pairwise comparison showed that the proportion of bold fish was lower at $8 \mathrm{dpf}(14.5 \%)$ than at $21 \mathrm{dpf}(35 \%)$ during the larval experiment ( $<$ 0.001). The proportion of bold fish at 21 dpf did not differ between the larval and juvenile experiments (35\% in the larval experiment and $48 \%$ in juvenile experiment; $\mathrm{p}=0.47$ ). Finally, the proportion of bold fish in the juvenile experiment did not differ between 21 and $60 \mathrm{dpf}$ (48\% at both ages; $\mathrm{p}=1$ ).

\section{3 | Behavioural consistency across context}

PCA axis 1 explained 69 and $75 \%$ of the variability observed in the behavioural responses at 8 and 21 dpf, respectively, during the larval experiment. PCA axis 1 explained 70 and 58\% of 
the variability observed in the behavioural responses at 21 and $60 \mathrm{dpf}$, respectively, during the juvenile experiment. PCA axis 1 was equally explained by the distance travelled $(\mathrm{cm})$ and the proportion of time spent in the peripheral/bottom area (\%) for each age group. Individuals with a high behavioural score expressed higher activity and higher thigmotaxis than individuals with low behavioural scores (Table 1).

During the larval experiment, PCA axis 1 scores were significantly higher for bold fish than shy fish at $8 \mathrm{dpf}(\mathrm{W}=1435, \mathrm{p}=0.04)$ and a "trend" was observed at $21 \mathrm{dpf}(\mathrm{W}=468, \mathrm{p}=$ 0.09). During the juvenile experiment, bold fish had higher behavioural scores than shy fish at both 21 and $60 \mathrm{dpf}(\mathrm{W}=1046, \mathrm{p}=0.04$ and $\mathrm{W}=374, \mathrm{p}=0.04$, respectively; Figure 5). This shows that bold and shy fish characterized using the risk-taking test differed in their behavioural responses during the open-field and novel tank-diving tests. Bold fish displayed higher activity and higher thigmotaxis than shy fish, both during the larval and juvenile experiments, except for a "trend" for $21 \mathrm{dpf}$ during the larval experiment.

\section{4 | Behavioural consistency over age}

We repeated the same procedure, marking either bold fish in some replicates ( $\mathrm{n}=3$ for both larval and juvenile experiments) or shy fish in others ( $n=2$ and $n=3$ replicates for the larval and juvenile experiments, respectively), to account for potential bias induced by marking. The data were pooled, as we observed no difference in survival or behavioural responses (see section "Validation of alizarin red S marking procedure"). Approximately 33\% of bold and $61 \%$ of shy fish expressed behavioural consistency during the risk taking-test between 8 and 21 dpf. Between 21 and 60 dpf, 41\% of bold and 61\% of shy fish showed behavioural consistency (Figure 6). The proportion of consistent behaviour did not differ from theoretical 
random probability for bold $(\mathrm{p}=0.12)$ or shy fish $(\mathrm{p}=0.28)$ between 8 and $21 \mathrm{dpf}$. Between 21 and $60 \mathrm{dpf}$, the proportion of consistent behaviour also did not differ from theoretical random probability for bold $(\mathrm{p}=0.52)$ or shy fish $(\mathrm{p}=0.40)$. However, shy fish tended to display more consistent behaviour than bold fish between 8 and $21 \mathrm{dpf}\left(\chi^{2}=3.5\right.$, df $=1, \mathrm{p}=$ 0.06). This trend disappeared between 21 and $60 \mathrm{dpf}\left(\chi^{2}=1.1, \mathrm{df}=1, \mathrm{p}=0.29\right.$; Figure 6).

\section{4 | DISCUSSION}

Coping style is assumed to be consistent across contexts and over time. However, this has been poorly studied during early life stages. In parallel to development, early experience can affect individual coping capacity, including in fishes (Colson et al, 2018; 2019; Vindas et al., 2019). In the present study, we aimed to evaluate coping style in fish, starting at the larval stage, and to evaluate the consistency of the coping style over age i.e. by comparing the results between the larval and juvenile stages. We thus conducted two distinct experiments, a larval experiment from 8 to $21 \mathrm{dpf}$ and a juvenile experiment from 21 to $60 \mathrm{dpf}$. Within each experiment, we repeated two behavioural tests (i.e. the group risk-taking test and the novel environment test) at the two start and end ages to evaluate both the consistency across contexts and over time.

The first step in both experiments was to evaluate boldness using the group risk-taking test. In the larval experiment, the proportion of bold larvae exiting the shelter was $14.5 \%$ of tested individuals at $8 \mathrm{dpf}$. This result contrasts with those of previous studies, which reported a proportion of more than $90 \%$ of larvae exiting the shelter (Tudorache et al., 2015). This 
difference may be due to the fish strain, as we used the TU strain, whereas previous studies used a cross between the AB and TL strains. Indeed, several studies have reported significant behavioural differences between classical wildtype zebrafish strains, such as AB and TL (van den Bos et al., 2017; Mustafa et al., 2019), including at early stages, as shown for AB and TU (Vignet et al., 2013). Another explanation for the difference may be the lighting level of the room. Indeed, the higher the difference in lighting between the sheltered and open areas, the higher the level of required boldness. Such a difference in response according to lighting level in a behavioural test has already been shown for zebrafish larvae (Schnorr et al., 2012). In the juvenile experiment, $48 \%$ of the individuals were characterized as bold at $21 \mathrm{dpf}$, which is slightly higher than that determined for zebrafish adults (26\%) by Rey et al. (2013). However, this proportion is in the range of what is generally observed during group risk-taking tests in other fish species (e.g. Castanheira et al., 2013; Alfonso et al., 2019; Carbonara et al., 2019). Overall, the results from the larval and juvenile experiments show that the proportion of bold fish in the zebrafish population increased from 8 to $21 \mathrm{dpf}$ (from 14.5 to $35-48 \%$, respectively) and was then stable, at least until $60 \mathrm{dpf}$. These results suggest that the proportion of fish with bold/shy traits is more variable during early life, before stabilizing around $21 \mathrm{dpf}$, at the time of mid-metamorphosis. Further experiments with closer timepoints will be needed to precisely quantify the time of stabilisation of the bold/shy proportion of the population.

In both experiments, the group risk-taking test was followed the day after by a novel environment test to evaluate consistency across contexts and to determine a behavioural syndrome. Bold fish in both the larval and juvenile experiments showed higher activity and 
higher thigmotaxis during the open-field and novel-tank diving tests, respectively. However, we observed weaker behavioural consistency across contexts at $21 \mathrm{dpf}$ in the larval experiment, suggesting that the group risk-taking test was less discriminant for this age group (i.e. higher proportion of 'false' bold fish). This could also be attributed to the previous experience and manipulation of the fish at $8 \mathrm{dpf}$ or smaller sample size at this experimental age. Such a behavioural syndrome, higher activity in bold individuals, is indeed well described in the literature, including in fish (Moretz et al., 2007; Réale et al., 2010; Geffroy et al., 2015; Castanheira et al., 2017), whereas the link between boldness and thigmotaxis is less well documented and still debated (Burns, 2008; Dahlbom et al., 2011; Ariyomo et al., 2013; Thörnqvist et al., 2019). In our study, bold fish showed higher thigmotaxis. Given that shy individuals are considered to be more plastic than bold ones (Ruiz-Gomez et al., 2008; Chapman et al., 2010; Coppens et al., 2010; Laubu et al., 2016), it is possible that shy fish may more easily adapt to the open field or a novel tank than bold fish, which could explain the lower thigmotaxis in shy fish. Another explanation could be that shy individuals display freezing behaviour in the centre of the arena following introduction into the novel environment. Overall, this consistency of behavioural responses across contexts allowed us to define exiting fish as proactive, whereas the others were defined as reactive individuals, regardless of life stage.

The same two tests were repeated 13 days later in the larval experiment and 40 days later in the juvenile experiment to evaluate consistency over time (i.e. across life stages). Overall, we observed no behavioural consistency between ages, either in the larval or juvenile experiments for bold or shy fish. This contrasts with strong behavioural consistency 
previously reported in zebrafish adults over a period of 10 months (Rey et al., 2013). Our results are however in accordance with those of other studies, which analysed consistency over time across different ontogenic stages. In nine-spine sticklebacks, starting at $30 \mathrm{dph}$, feeding behaviour was shown to be consistent within ontogenic stages (juveniles or adults) but not across stages (Herczeg et al., 2013). A similar result was obtained with Eastern mosquitofish (Gambusia holbrooki), which displayed very little consistency of personality across ontogenic stages (Polverino et al., 2016). The same inconsistency of individual behaviour has been shown for three-spine sticklebacks Gasterosteus aculeatus during the juvenile, sub-adult, and adult stages (Bell and Stamps, 2004) and for sea bream Sparus aurata between the juvenile and adult stages (Castanheira et al., 2016). Finally, the only other study that follows behavioural consistency from the larval to juvenile stages also concluded that there was no consistency between the two life stages in mangrove killifish (Edenbrow and Croft, 2011); the onset of behavioural consistency occurring near sexual maturity during the juvenile stage. However, in this study, the fish were raised in isolation, which can introduce biases, including individual stress and/or divergence in physicochemical rearing conditions. In addition, the authors also reported the absence of cross-context consistency before this same age. This is consistent with the fact that no evidence for personality was found in 30-day-old juvenile Eastern mosquitofish versus sub-adults and adults (60 and 120 dph, Polverino et al. (2016)). Our work, however, leads to a different conclusion, as we indeed observed consistency across contexts at all studied ages, with a clear behavioural syndrome already visible at the larval stage. This is in accordance with a report showing that 8 dpf zebrafish larvae exhibit a coping style (Tudorache et al., 2015). Overall, our results suggest that 
important changes that occur in the organism during transition between life stages may trigger shifts in coping styles. Metamorphosis is a complex process that includes several morphological and physiological changes that are more-or-less synchronised. Further, and beyond metamorphosis, experience and learning capabilities also mature and it has been shown that both classical and operant learning become efficient at approximately three weeks of age (Valente et al., 2012). When comparing results between 8 and $21 \mathrm{dpf}$ (Larval experiment) or 21 and $60 \mathrm{dpf}$ (Juvenile experiment), we did not find behavioural consistency between the successive ages. These suggest that during all the larval stage, the continuous biological processes that shape the morphological and physiological changes of fish between the two stages, also modify the personality that will become consistent later during zebrafish life. Further studies are needed to refine the time window of the onset of stable personality traits in zebrafish. This could probably be near sexual maturity, as found by Edenbrow and Croft (2011) for mangrove killifish.

In addition, we used alizarin red S to mark the fish and thereby mark them between the two successive ages of each experiment. The marks were visible in the zebrafish bones and otoliths at least 39 days after marking. Alizarin red S marking (at the concentration chosen) did not induce particular mortality or developmental defects in zebrafish, as reported in previous studies with other fish species (Caraguel et al., 2015; Lü et al., 2015; Stanczak et al., 2015). Marking did not induce behavioural disruption in larvae, as shown by the LPMR test, or a differential response after determination of the coping style. These results thus validate alizarin red S for non-invasive marking, suitable for individual or group identification starting 
at early life stages in fish. The use of alizarin red S provides new perspectives for the further study of the longitudinal evolution of various traits, including behaviour, across life stages.

In conclusion, the occurrence of boldness in zebrafish, as measured using the group risktaking test, is lower at $8 \mathrm{dpf}$ than at 21 and $60 \mathrm{dpf}$. Fish categorized as bold or shy using the group risk-taking test display differential behavioural responses in the open-field or noveltank diving tests at 8, 21, and 60 dpf. Bold fish are more active and show higher thigmotaxis than shy fish, regardless of age, starting at least at $8 \mathrm{dpf}$. There was no behavioural consistency however between different ages which suggests behavioural plasticity across life stages. Coping styles are thus present at the larval stage but are not fixed and may evolve though individual experience and physiological changes (Castanheira et al., 2016; Ferrari et al., 2016).

\section{ACKNOWLEDGMENTS}

We thank Christine Jarc, Didier Leguay, and Lucette Joassard from Ifremer (LRH) for their valuable help in running this experiment. We also thank anonymous reviewers for helpful comment on a previous version of the manuscript.

\section{CONTRIBUTION}

S.A conceptualized the experiment and methodology, performed the experiments, analysed the data, wrote the original draft, and reviewed and edited the manuscript. M.P performed the 
experiments and analysed the data. M.L.B and X.C acquired the funding, conceptualized the experiment and methodology, supervised the experiments and data analyses, reviewed and edited the manuscript.

\section{REFERENCES}

Ahmad, F. \& Richardson, M. K. (2013). Exploratory behaviour in the open field test adapted for larval zebrafish: impact of environmental complexity. Behav Processes 92, 88-98.

Alfonso, S., Blanc, M., Joassard, L., Keiter, S. H., Munschy, C., Loizeau, V., Begout, M. L. \& Cousin, X. (2019a). Examining multi- and transgenerational behavioral and molecular alterations resulting from parental exposure to an environmental PCB and PBDE mixture. Aquat Toxicol 208, 29-38.

Alfonso, S., Sadoul, B., Gesto, M., Joassard, L., Chatain, B., Geffroy, B. \& Begout, M. L. (2019b). Coping styles in European sea bass: The link between boldness, stress response and neurogenesis. Physiol Behav 207, 76-85.

Ariyomo, T. O., Carter, M. \& Watt, P. J. (2013). Heritability of boldness and aggressiveness in the zebrafish. Behav Genet 43, 161-167. 
Bell, A. M. \& Stamps, J. A. (2004). Development of behavioural differences between individuals and populations of sticklebacks, Gasterosteus aculeatus. Animal Behaviour 68, $1339-1348$.

Bensimon-Brito, A., Cardeira, J., Dionísio, G., Huysseune, A., Cancela, M. L. \& Witten, P. E. (2016). Revisiting in vivo staining with alizarin red S - a valuable approach to analyse zebrafish skeletal mineralization during development and regeneration. BMC Developmental Biology 16, 2.

Burns, J. G. (2008). The Validity of Three Tests of Temperament in Guppies (Poecilia reticulata). Journal of Comparative Psychology 122, 344-356.

Caraguel, J. M., Charrier, F., Mazel, V. \& Feunteun, E. (2015). Mass marking of stocked European glass eels (Anguilla anguilla) with alizarin red S. Ecology of Freshwater FIsh 24, 435-442.

Carbonara, P., Alfonso, S., Zupa, W., Manfrin, A., Fiocchi, E., Pretto, T., Spedicato, M.T. \& Lembo, G. (2019). Behavioral and physiological responses to stocking density in sea bream (Sparus aurata): Do coping styles matter? Physiol Behav 212, 112698.

Castanheira, M. F., Conceição, L. E. C., Millot, S., Rey, S., Bégout, M. L., Damsård, B., Kristiansen, T., E, H., Øverli, Ø. \& Martins, C. I. M. (2017). Coping styles in farmed fish: consequences for aquaculture. Reviews in Aquaculture 9, 23-41. 
Castanheira, M. F., Herrera, M., Costas, B., Conceicao, L. E. C. \& Martins, C. I. M. (2013). Can We Predict Personality in Fish? Searching for Consistency over Time and across Contexts. PLoS ONE 8, e62037.

Castanheira, M. F., Martinez Paramo, S., Figueiredo, F., Cerqueira, M., Millot, S., Oliveira, C. C., Martins, C. I. \& Conceicao, L. E. (2016). Are coping styles consistent in the teleost fish Sparus aurata through sexual maturation and sex reversal? Fish Physiol Biochem 42, 1441-1452.

Chapman, B. B., Morrell, L. J. \& Krause, J. (2010). Unpredictability in food supply during early life influences boldness in fish. Behavioral Ecology 21, 501-506.

Coppens, C. M., de Boer, S. F. \& Koolhaas, J. M. (2010). Coping styles and behavioural flexibility: towards underlying mechanisms. Philosophical Transactions of the Royal Society B: Biological Sciences 365, 4021-4028.

Dahlbom, S. J., Lagman, D., Lundstedt-Enkel, K., Sundstrom, L. F. \& Winberg, S. (2011). Boldness Predicts Social Status in Zebrafish (Danio rerio). Plos One 6.

DeLaurier, A., Eames, B. F., Blanco-Sanchez, B., Peng, G., He, X., Swartz, M. E., Ullmann, B., Westerfield, M. \& Kimmel, C. B. (2010). Zebrafish sp7:EGFP: A transgenic for studying otic vesicle formation, skeletogenesis, and bone regeneration. Genesis 48, 505-511. 
Dray, S. \& Dufour, A. B. (2007). The ade4 package: implementing the duality diagram for ecologists. Journal of Statistical Software 22, 1-20.

Edenbrow, M. \& Croft, D. P. (2011). Behavioural types and life history strategies during ontogeny in the mangrove killifish, Kryptolebias marmoratus. Animal behaviour 82(4), 731-741.

Egan, R. J., Bergner, C. L., Hart, P. C., Cachat, J. M., Canavello, P. R., Elegante, M. F., Elkhayat, S. I., Bartels, B. K., Tien, A. K., Tien, D. H., Mohnot, S., Beeson, E., Glasgow, E., Amri, H., Zukowska, Z. \& Kalueff, A. V. (2009). Understanding behavioral and physiological phenotypes of stress and anxiety in zebrafish. Behav Brain Res 205, 38-44.

Ferrari, S., Leguay, D., Vergnet, A., Vidal, M. O., Chatain, B. \& Begout, M. L. (2016). Unpredictability in food supply during early life influences growth and boldness in European seabass, Dicentrarchus labrax (vol 180, pg 147, 2016). Applied Animal Behaviour Science 185, 152-152.

Ferrari, S., Millot, S., Leguay, D., Chatain, B. \& Bégout, M. L. (2015). Consistency in European seabass coping styles: A life-history approach. Applied Animal Behaviour Science 167, 74-88. 
Geffroy, B., Sadoul, B. \& Bardonnet, A. (2015). Behavioural syndrome in juvenile eels and its ecological implications. Behaviour 152, 147-166.

Gostling, S. D. (2001). From mice to men: What can we learn about personality from animal research. Psycological Bulletin 127, 45-86.

Hale, M. E. (2014). Developmental change in the function of movement systems: transition of the pectoral fins between respiratory and locomotor roles in zebrafish. Integr Comp Biol 54, 238-249.

Herczeg, G., Ab Ghani, N. I. \& Merila, J. (2013). Evolution of stickleback feeding behaviour: genetics of population divergence at different ontogenetic stages. Journal of Evolutionary Biology 26, 955-962.

Huntingford, F. A., Andrew, G., Mackenzie, S., Morera, D., Coyle, S. M., Pilarczyk, M. \& Kadri, S. (2010). Coping strategies in a strongly schooling fish, the common carp Cyprinus carpio. J Fish Biol 76, 1576-1591.

Koolhaas, J. M., Korte, S. M., De Boer, S. F., Van Der Vegt, B. J., Van Reenen, C. G., Hopster, H., De Jong, I. C., Ruis, M. A. \& Blokhuis, H. J. (1999). Coping styles in animals: current status in behavior and stress-physiology. Neurosci Biobehav Rev 23, 925-935. 
Laubu, C., Dechaume-Moncharmont, F. X., Motreuil, S. \& Schweitzer, C. (2016). Mismatched partners that achieve postpairing behavioral similarity improve their reproductive success. Sci Adv 2, e1501013.

Lawrence, C. (2007). The husbandry of zebrafish (Danio rerio): A review. Aquaculture 269, $1-20$.

Lü, H. J., Fu, M., Xi, D., Yao, W. Z., Su, S. Q. \& Wu, Z. L. (2015). Experimental evaluation of using calcein and alizarin red S for immersion marking of bighead carp Aristichthys nobilis (Richardson, 1845) to assess growth and identification of marks in otoliths, scales and fin rays. Journal of Apllied Ichthyology 31, 665-674.

Millot, S., Bégout, M.-L. \& Chatain, B. (2009). Exploration behaviour and flight response toward a stimulus in three sea bass strains (Dicentrarchus labrax L.). Applied Animal Behaviour Science 119, 108-114.

Millot, S., Cerqueira, M., Castanheira, M. F., Øverli, Ø., Oliveira, R. F. \& Martins, C. I. (2014). Behavioural stress responses predict environmental perception in European sea bass (Dicentrarchus labrax). PLoS One 9, e108800.

Moretz, J. A., Martins, A. P. \& Robison, B. D. (2007). Behavioral syndromes and the evolution of correlated behavior in zebrafish. Behavioral Ecology 18, 556-562. 
Mustafa, A., Roman, E. \& Winberg, S. (2019). Boldness in Male and Female Zebrafish

(Danio $\quad$ rerio) Is Dependent on Strain and Test. Front Behav Neurosci 13, 248.

Nüsslein-Volhard, C. \& Dahm, R. (2002). Zebrafish. A Practical Approach. Oxford Univ. Press, 303 pages.

Orban, L., Sreenivasan, R. \& Olsson, P.-E. (2009). Long and winding roads: Testis differentiation in zebrafish. Molecular and Cellular Endocrinology 312, 35-41.

Øverli, Ø., Korzan, W. J., Hoglund, E., Winberg, S., Bollig, H., Watt, M., Forster, G. L., Barton, B. A., E, O. V., Renner, K. J. \& Summers, C. H. (2004). Stress coping style predicts aggression and social dominance in rainbow trout. Horm Behav 45, 235-241.

Øverli, Ø., Pottinger, T. G., Carrick, T. R., Øverli, E. \& Winberg, S. (2002). Differences in behaviour between rainbow trout selected for high- and low-stress responsiveness. Journal of Experimental Biology 205, 391-395.

Øverli, Ø., Sorensen, C. \& Nilsson, G. E. (2006). Behavioral indicators of stress-coping style in rainbow trout: Do males and females react differently to novelty? Physiol Behav 87, 506-512.

Øverli, Ø., Sorensen, C., Pulman, K. G., Pottinger, T. G., Korzan, W., Summers, C. H. \& Nilsson, G. E. (2007). Evolutionary background for stress-coping styles: relationships 
between physiological, behavioral, and cognitive traits in non-mammalian vertebrates.

Neurosci Biobehav Rev 31, 396-412.

Pagnussat, N., Piato, A. L., Schaefer, I. C., Blank, M., Tamborski, A. R., Guerim, L. D., Bonan, C. D., Vianna, M. R. M. \& Lara, D. R. (2013). One for All and All for One: The Importance of Shoaling on Behavioral and Stress Responses in Zebrafish. Zebrafish 10, 338-342.

Parichy, D. M., Elizondo, M.R., Mills, M.G., Gordon, T.N. \& Engeszer R.E. (2009). Normal table of postembryonic zebrafish development: Staging by externally visible anatomy of the living fish. Dev Dyn 238, 2975-3015.

Parichy, D. M. \& Turner, J. M. (2003). Zebrafish puma mutant decouples pigment pattern and somatic metamorphosis. Dev Biol 256, 242-257.

Pasquet, A., Sebastian, A., Bégout, M. L., LeDore, Y., Teletchea, F. \& Fontaine, P. (2016). First insight into personality traits in Northern pike (Esox lucius) larvae: a basis for behavioural studies of early life stages. Environmental Biology Fishes 99, 105-115.

Poisson, A., Valotaire, C., Borel, F., Bertin, A., Darmaillacq, A.S., Dickel, L. \& Colson, V. (2018). Embryonic exposure to a conspecific alarm cue triggers behavioural plasticity in juvenile rainbow trout. Anim behav 133, 35-45. 
Polverino, G., Cigliano, C., Nakayama, S. \& Mehner, T. (2016). Emergence and development of personality over the ontogeny of fish in absence of environmental stress factors. Behavioral Ecology and Sociobiology 70, 2027-2037.

R Core Team. (2013). R: A language and environment for statistical computing. $R$ Foundation for Statistical Computing, Vienna, Austria.

Reale, D., Garant, D., Humphries, M. M., Bergeron, P., Careau, V. \& Montiglio, P. O. (2010). Personality and the emergence of the pace-of-life syndrome concept at the population level. Philos Trans R Soc Lond B Biol Sci 365, 4051-4063.

Rey, S., Boltana, S., Vargas, R., Roher, N. \& Mackenzie, S. (2013). Combining animal personalities with transcriptomics resolves individual variation within a wild-type zebrafish population and identifies underpinning molecular differences in brain function. Mol Ecol 22, 6100-6115.

Ruiz-Gomez, M. d. L., Kittilsen, S., Höglund, E., Huntingford, F. A., Sørensen, C., Pottinger, T. G., Bakken, M., Winberg, S., Korzan, W. J. \& Øverli, Ø. (2008). Behavioral plasticity in rainbow trout (Oncorhynchus mykiss) with divergent coping styles: When doves become hawks. Hormones and Behavior 54, 534-538.

Schnorr, S. J., Steenbergen, P. J., Richardson, M. K. \& Champagne, D. L. (2012). Measuring thigmotaxis in larval zebrafish. Behaviour Brain Research 228, 367-374. 
Sih, A., Bell, A. \& Chadwick Johnson, J. (2004). Behavioral syndromes: an ecological and evolutionary overview. Trends in Ecology \& Evolution 19, 372-378.

Singleman, C., \& Holtzman, N. G. (2014). Growth and Maturation in the Zebrafish, Danio Rerio: A Staging Tool for Teaching and Research. Zebrafish, 11, 396-406.

Stanczak, K., Krejszeff, S., Debowska, M., Mierzejewska, K., Wozniak, M. \& Hliwa, P. (2015). Mass marking of Leuciscus idus larvae using Artemia salina as a vector of fluorescent dyes. J Fish Biol 87, 799-804.

Toms, C. N. \& Echevarria, D. J. (2014). Back to basics: Searching for a comprehensive framework for exploring individual differences in zebrafish (Danio Rerio) behavior. Zebrafish 11.

Thörnqvist, P.O., McCarrick, S., Ericsson, M., Roman, E., \& Winberg, S. (2019). Bold zebrafish (Danio rerio) express higher levels of delta opioid and dopamine D2 receptors in the brain compared to shy fish. Behav Brain res 359, 927-934.

Tu, S. \& Johnson, S. L. (2011). Fate restriction in the growing and regenerating zebrafish fin. Developmental Cell 20, 725-732.

Tudorache, C., Schaaf, M. J. \& Slabbekoorn, H. (2013). Covariation between behaviour and physiology indicators of coping style in zebrafish (Danio rerio). J Endocrinol 219, 251-258. 
Tudorache, C., ter Braake, A., Tromp, M., Slabbekoorn, H. \& Schaaf, M. J. (2015). Behavioral and physiological indicators of stress coping styles in larval zebrafish. Stress 18, 121-128.

Valente, A., Huang, K. H., Portugues, R. \& Engert, F. (2012). Ontogeny of classical and operant learning behaviors in zebrafish. Learn Mem 19, 170-177.

van den Bos, R., Mes, W., Galligani, P., Heil, A., Zethof, J., Flik, G. \& Gorissen, M. (2017). Further characterisation of differences between TL and AB zebrafish (Danio rerio): Gene expression, physiology and behaviour at day 5 of the larval stage. Plos One 12.

Vignet, C., Begout, M. L., Pean, S., Lyphout, L., Leguay, D. \& Cousin, X. (2013). Systematic screening of behavioral responses in two zebrafish strains. Zebrafish 10, 365-375.

Vignet, C., Joassard, L., Lyphout, L., Guionnet, T., Goubeau, M., Le Menach, K., Brion, F., Kah, O., Chung, B. C., Budzinski, H., Begout, M. L. \& Cousin, X. (2015). Exposures of zebrafish through diet to three environmentally relevant mixtures of PAHs produce behavioral disruptions in unexposed F1 and F2 descendant. Environ Sci Pollut Res Int 22, 16371-16383.

Vindas, M.A., Fokos, S., Pavlidis, M., Höglund, E., Dionysopoulou, S., Ebbesson L.O.E., Papandroulakis, N. \& Dermon C.R (2019). Early life stress induces long-term changes 
in limbic areas of a teleost fish: the role of catecholamine systems in stress coping. Sci rep 8, 5638.

Westerfield, M. (2000). The zebrafish book. A guide for the laboratory use of zebrafish (Danio rerio). 4th ed., Univ. of Oregon Press, Eugene.

Wong, R. Y., French, J. \& Russ, J. B. (2019). Differences in stress reactivity between zebrafish with alternative stress coping styles. R Soc Open Sci 6, 181797. 


\section{Significance statement:}

Personality traits are considered as consistent across context and over time but little is known about consistency over life stages. This study reports personality traits consistency when evaluated in different contexts at larval or juvenile stages but not between stages revealing that personality can evolve after full metamorphosis. This study was made possible using a vital staining and brings new perspectives to further study longitudinal evolution of various traits over life stages in fishes.

This article has been accepted for publication and undergone full peer review but has not been through the copyediting, typesetting, pagination and proofreading process which may lead to differences between this version and the Version of Record. Please cite this article as doi: $10.1111 / \mathrm{jfb} .14310$ 

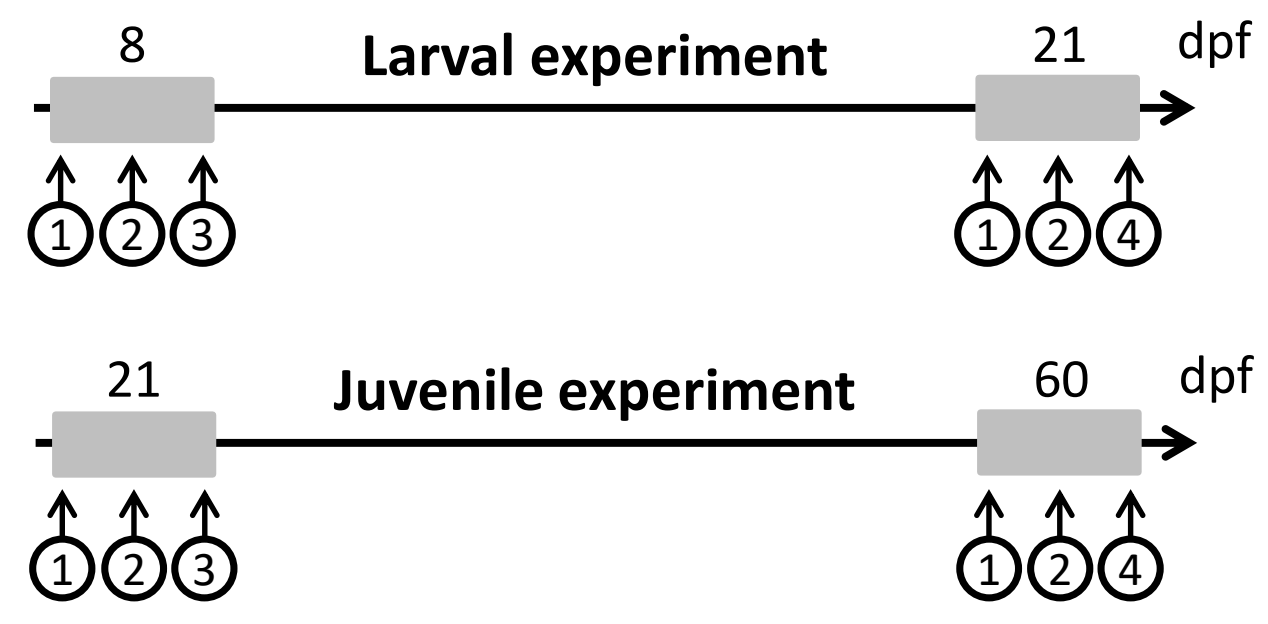

- Legend:

Experimental procedure period

(1) Risk taking test

(2) Novel environment test

(3) Marking using alizarin red $\mathrm{S}$

(4) Reading of alizarin red S staining 

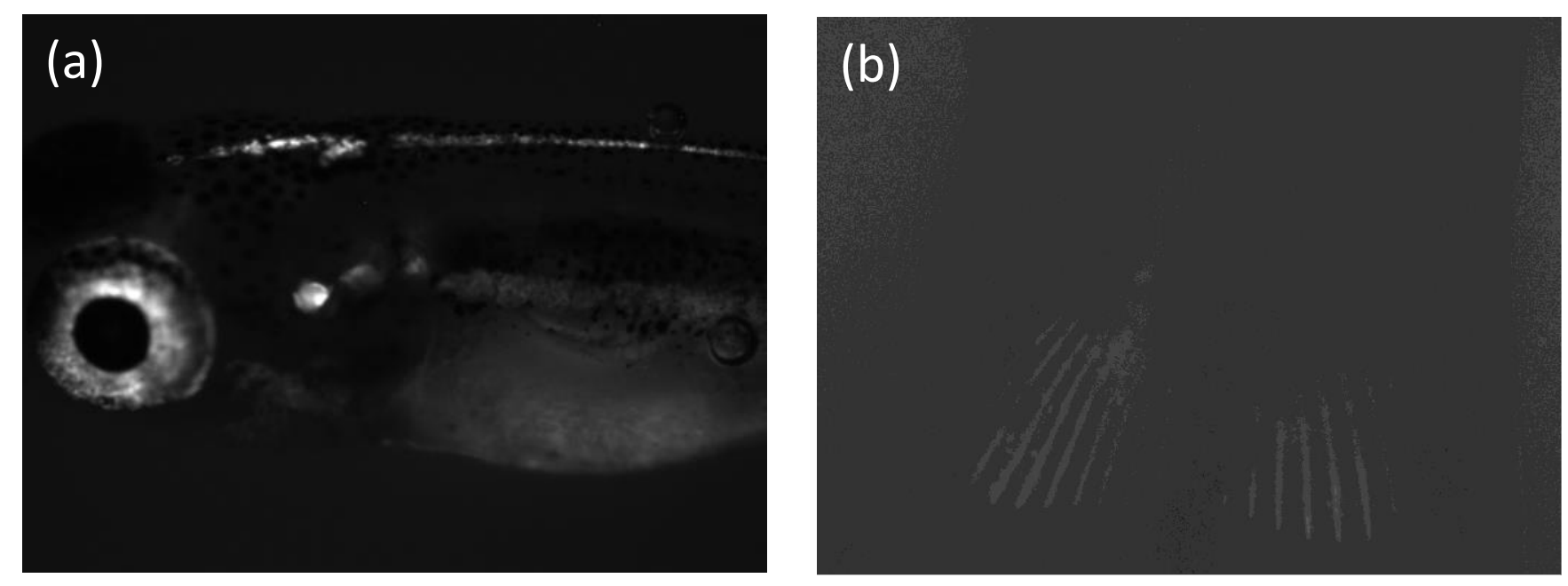

This article is protected by copyright. All rights reserved. 


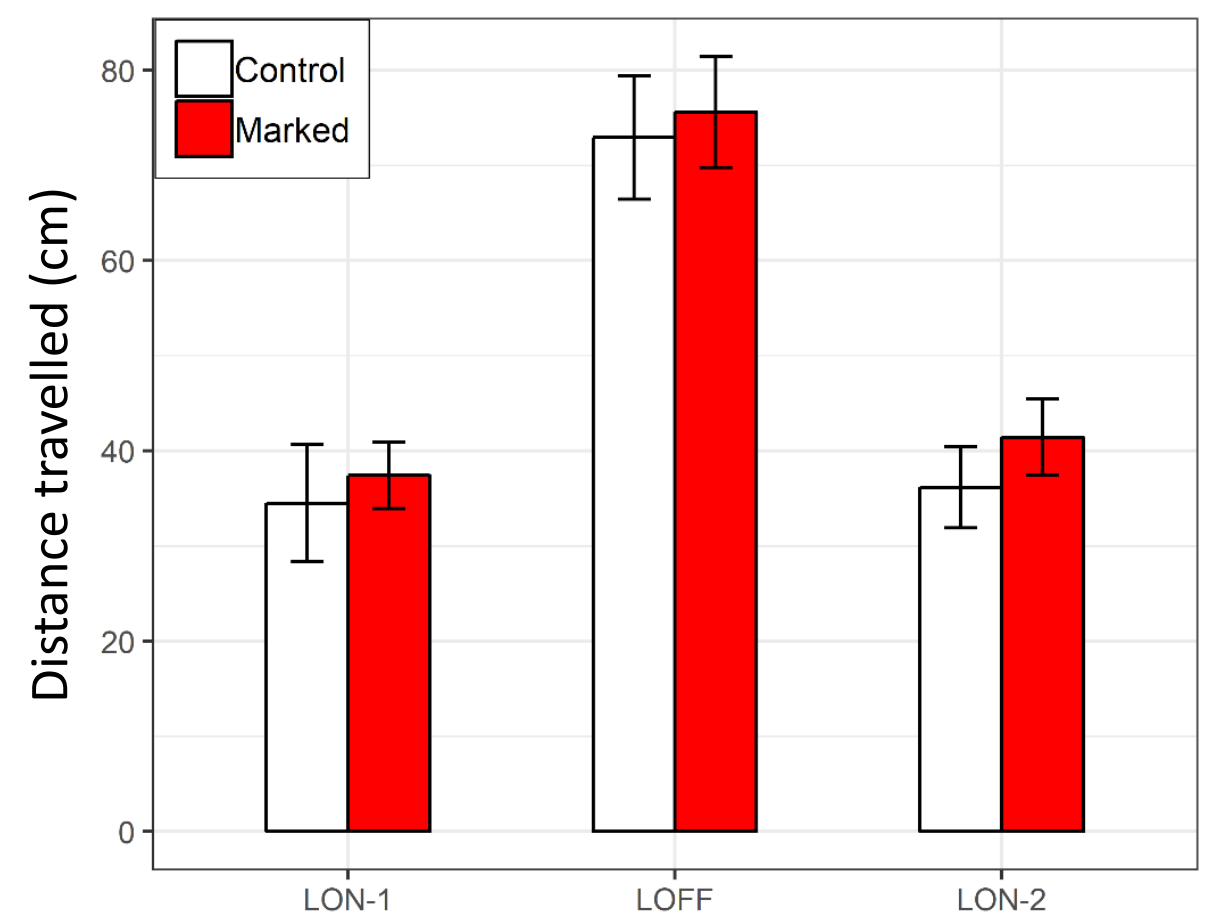

This article is protected by copyright. All rights reserved. 
(a) Larval experiment

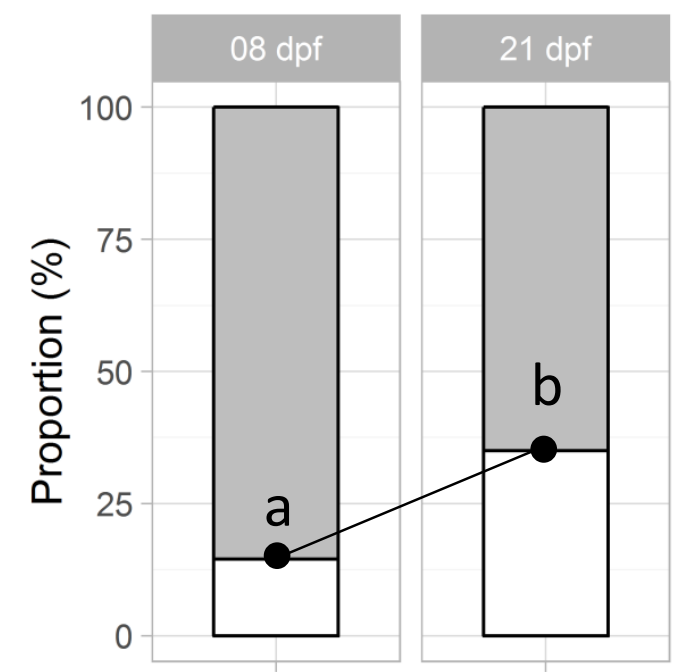

(b) Juvenile experiment

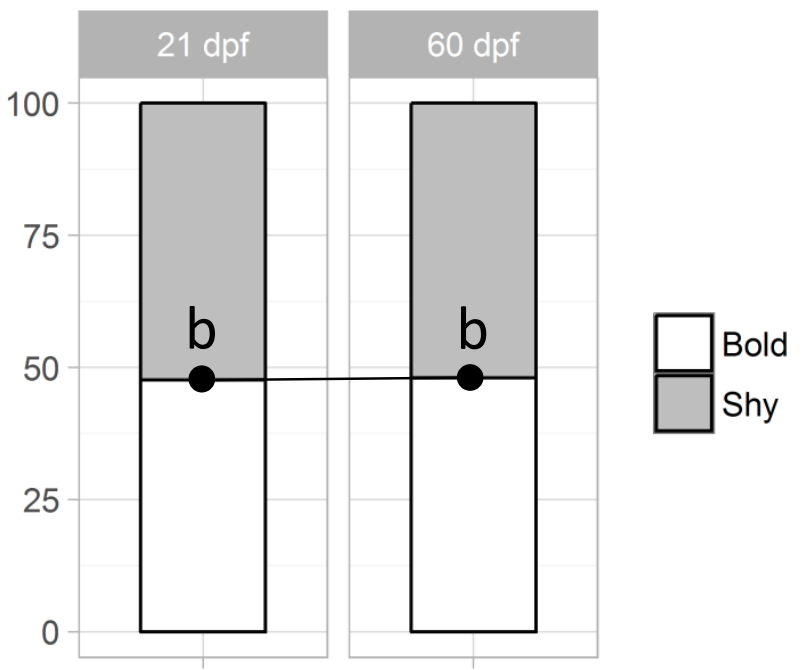

This article is protected by copyright. All rights reserved. 
(a) Larval experiment

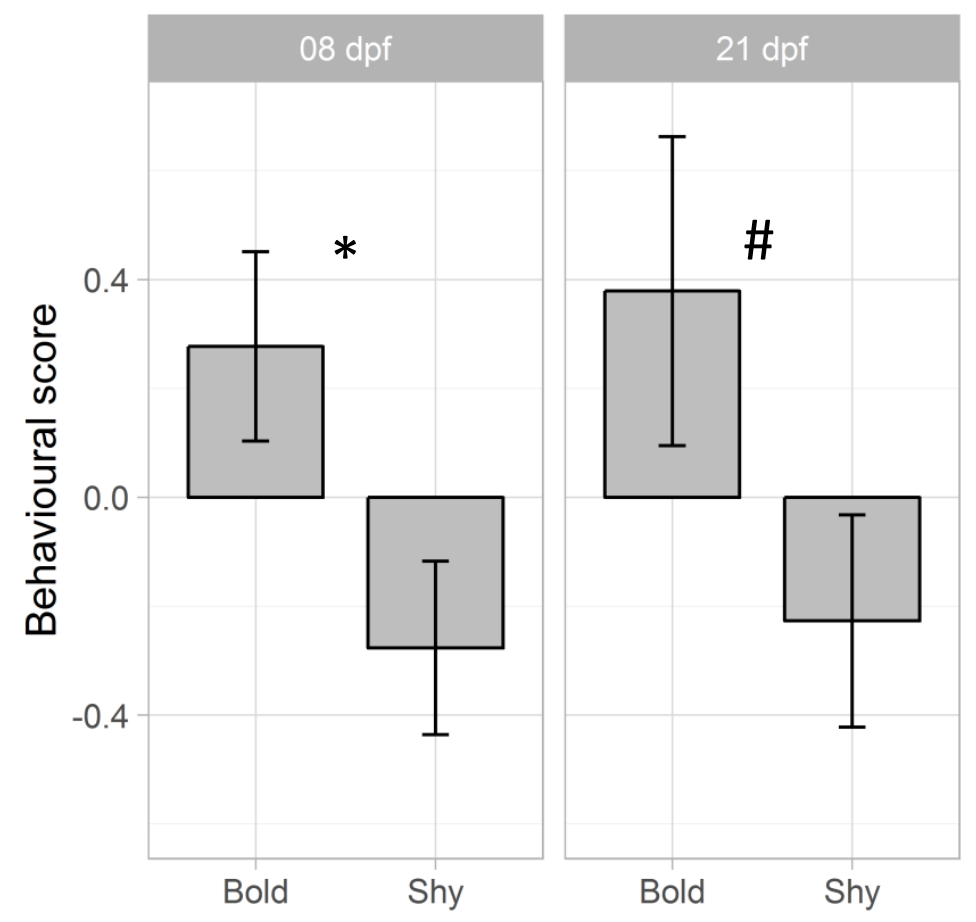

(b) Juvenile experiment

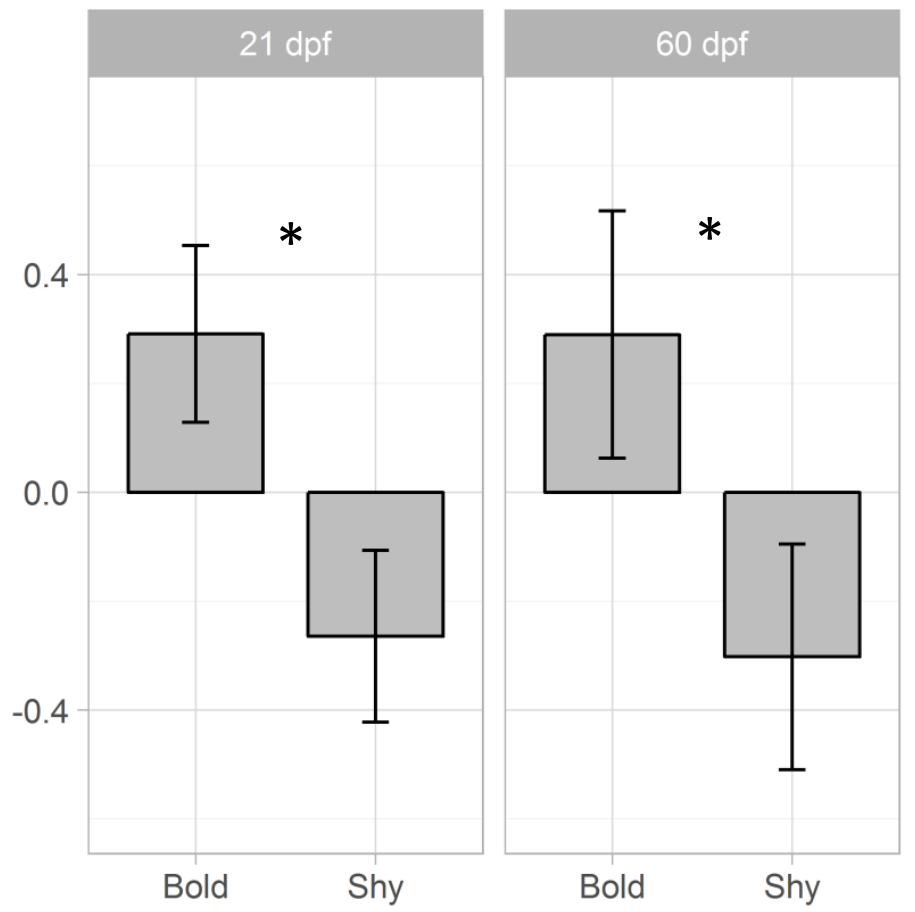

This article is protected by copyright. All rights reserved. 


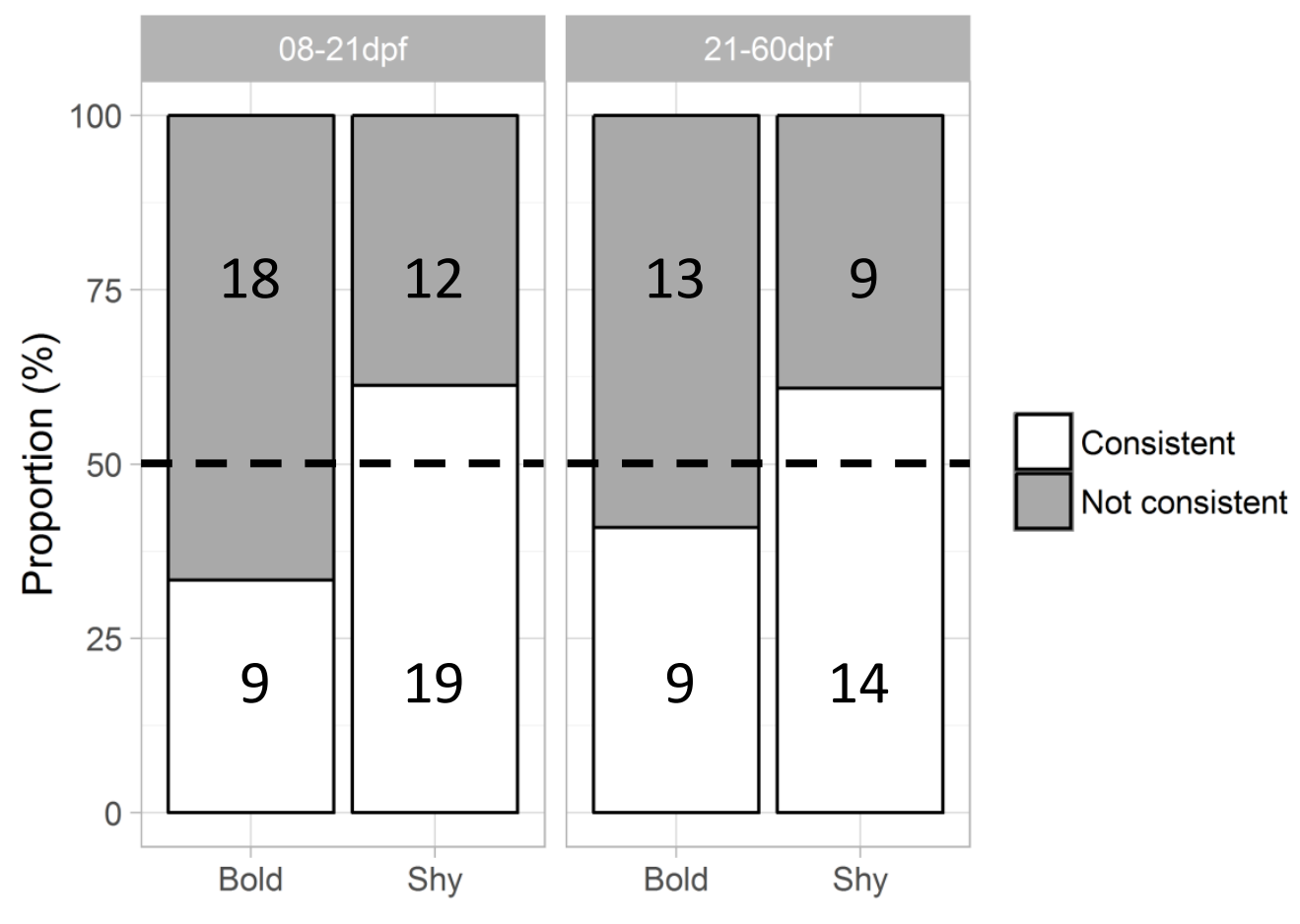

This article is protected by copyright. All rights reserved. 
TABLE 1. Loadings of behavioural variables for the PCA axis 1 performed during larval experiment ( 8 and $21 \mathrm{dpf}$ ) and juvenile experiment (21 and $60 \mathrm{dpf}$ ).

Larval experiment Juvenile experiment

\begin{tabular}{ccccc}
\hline Variables in PCA axis 1 & $8 \mathrm{dpf}$ & $21 \mathrm{dpf}$ & $21 \mathrm{dpf}$ & $60 \mathrm{dpf}$ \\
\hline $\begin{array}{c}\text { Distance travelled (cm) } \\
\text { Proportion of time (\%) spent in periphery } \\
(8 \& \text { 21 dpf) or bottom area (60 dpf) }\end{array}$ & 0.83 & 0.87 & 0.84 & 0.76 \\
\hline Variance explained (\%) & 69 & 75 & 70 & 58 \\
\hline
\end{tabular}

This article is protected by copyright. All rights reserved. 
FIGURE 1. Graphical protocol used to monitor behavioural consistency across context and over time from larval to juvenile stages using the two different experiments. (1) Group risktaking test for boldness screening; (2) Individual behavioural characterization using either open field test (at 8 and $21 \mathrm{dpf}$ ) or novel tank diving test (at $60 \mathrm{dpf}$ ); (3) Marking using alizarin red S; (4) Reading of alizarin red S staining.

FIGURE 2. Example of alizarin red S marks observed in anaesthetised individuals in (a) otolith at $21 \mathrm{dpf}$ and (b) in caudal peduncle rays at $60 \mathrm{dpf}$.

FIGURE 3. Distance travelled during each 5-min steps (LON-1, LOFF, LON-2) of the LPMR performed at $9 \mathrm{dpf}$ for Control ( $n=30$, white bars) and marked larvae ( $n=30$, red bars). Values are mean \pm SE.

FIGURE 4. Proportion of bold (white bars) and shy (dark grey bars) individuals during risktaking test for (A) Larval experiment ( 8 dpf: $n=48$ bold, $n=282$ shy; 21 dpf: $n=21$ bold, $n=39$ shy) and (B) Juvenile experiment ( 21 dpf: $n=50$ bold, $n=55$ shy; 60 dpf: $n=24$ bold, $n=26$ shy). Pairwise comparison of proportions: different letters indicate significant difference for proportions of proactive fish across ages. Values are mean $\pm \mathrm{SE}$.

FIGURE 5. Individual behavioural scores (PCA axis 1) of bold and shy fish for (A) Larval experiment ( 8 dpf: $n=48$ bold, $n=48$ shy; 21 dpf: $n=21$ bold, $n=35$ shy) and (B) Juvenile 
experiment ( 21 dpf: $n=50$ bold, $n=55$ shy; 60 dpf: $n=24$ bold, $n=23$ shy). Wilcoxon sum rank test between coping styles within age \#: $p<0.10$ and $*$ : $p<0.05$. Values are mean \pm SE.

FIGURE 6. Proportion of fish with consistent (white bars) and non-consistent (dark grey bars) coping style during risk-taking test for each age in bold and shy fish (8-21 dpf: $n=27$ bold, $\mathrm{n}=31$ shy; 21-60 dpf: $\mathrm{n}=22$ bold, $\mathrm{n}=23$ shy). Dashed black line represents random theoretical probability to be categorized as bold or shy in the following risk-taking test. Values are mean $\pm \mathrm{SE}$. 